\title{
SENSIBILIZACIÓN A BISFENOL A Y BISFENOL F EN TRABAJADORES EXPUESTOS A RESINAS EPOXI
}

\author{
JIMÉNEZ BAJO, L.; FERNÁNDEZ GUARINO, M.; DEL POZO POZO, A. I.; \\ MARTINEZ-AMO GÁMEZ, J. L.; HERAS MENDAZA, F.; CONDE-SALAZAR GÓMEZ, L.
}

Servicio de Dermatología Laboral.

Escuela Nacional de Medicina del Trabajo.

Instituto de Salud Carlos III. Madrid.

\section{RESUMEN}

Las aplicaciones de las diferentes resinas epoxi basadas en bisfenol A y F son extensas. Las resinas epoxi basadas en bisfenol $\mathrm{F}$ son más nuevas y resistentes que las de bisfenol $\mathrm{A}$, y ambas son causa conocida de dermatitis de contacto alérgica.

Se estudian 39 casos de sensibilización a resinas epoxi en los últimos 5 años.

Los resultados obtenidos son: prevalencia de sensibilización a resinas epoxi entre los pacientes estudiados del $2 \%, 27$ varones y 8 mujeres, con una edad media de 42.77, un período de medio sensibilización de 23,8 meses. La sensibilización a bisfenol $\mathrm{F}$ desde que se incluye para las pruebas del parche en la batería de resinas epoxi es del $100 \%$ para los casos sensibilizados a la resina. La localización mayoritaria se dio en las manos, con una relevancia actual del $84 \%$. Se recomendó un cambio de puesto en el trabajo al $46 \%$ de estos pacientes.

El aumento de la sensibilización encontrada a bisfenol $\mathrm{F}$ puede explicarse por una sensibilización concomitante con bisfenol A, una declaración incorrecta de la composición de la resina o una reactividad cruzada de ambos.

\section{PALABRAS CLAVES}

Resina epoxi, bisfenol A, bisfenol F, sensibilización concomitante, reactividad cruzada.

\begin{abstract}
The applications of epoxy resins based on bisphenol $\mathrm{A}$ and $\mathrm{F}$ are extensive. Epoxy resins based on bisphenol $\mathrm{F}$ are new and more resistant than epoxy resins based on bisphenol A. Both of them cause allergic contact dermatitis.

In the last 5 years, we have studied 39 cases of sensitization to epoxy resin.

The results of our study were these: the global prevalence of epoxy resin sensitization was $2 \%$, 27 men and 8 women. The mean age was 42.77 years and the sensitization period was 23.8 months. The most frequent localization of the lesions were hands ( $84 \%$ of the patients). Since bisphenol $\mathrm{F}$ was included in epoxy resin battery for patch testing, a $100 \%$ of positives responses in patients sensitized to epoxy resin have been found. Sensitization to epoxy resins was a cause of change in workplace in $46 \%$ of cases in our series. There has been an increase in sensitization of bisphenol $\mathrm{F}$ that could be explained by a concomitant sensitization with bisphenol A, cross-reactivity between bisphenol $\mathrm{A}$ and $\mathrm{F}$ or because an incorrect chemical compound information of the resin.
\end{abstract}

\section{KEY WORDS}

Epoxy resin, bisphenol F, bisphenol A, concomitant sensitization, cross-reactivity. 


\section{INTRODUCCIÓN}

Las aplicaciones de las resinas epoxi son tan extensas y variadas debido a sus propiedades de resistencia, poder adhesivo, capacidad aislante y rapidez de manufactura ${ }^{(1)}$, de tanto interés en la industria (tabla I). Esto ha favorecido su vertiginoso desarrollo y la mejora de estas propiedades en cada nuevo producto lanzado al mercado.

\section{Tabla I. Algunas aplicaciones de la resina epoxi.}

- Bricolaje

- Construcción civil y militar: pinturas, suelos industriales, reparaciones.

- Material deportivo: skis, raquetas

- Medicina: marcapasos, prótesis.

- Metales: recubrimientos.

- Pegamentos

- Refuerzos de plásticos: fibra de vidrio, carbono y nylon.

Las excelentes propiedades de las resinas epoxi tienen un lado amargo, que es su gran poder de sensibilización ${ }^{(2)}$ y de desarrollo de dermatitis de contacto en trabajadores expuestos.

En la actualidad el uso de resina epoxi no queda restringido al ámbito laboral. Cada vez son más los productos utilizados de forma no profesional ${ }^{(2)}$, donde existe menor control.

Químicamente las resinas epoxi se forman a partir de policondensación de compuestos que poseen al menos dos átomos reactivos de hidrógeno (compuesto polihidroxilado) con epicloridrina. La gran mayoría de las resinas epoxi son diglicidil éteres de bisfenol A (DGEBA), aunque debido al interés por mejorar las características de estas resinas se han desarrollado otros compuestos, entre los que se encuentran las resinas de diglicidil éteres de bisfenol F (DGEBF), entre otros, aportando mayor resistencia, debido a su menor viscosidad ${ }^{(3)}$. El extendido uso de este compuesto hizo que se incluyera en la batería epoxi de pruebas epicutáneas en nuestro Servicio de Dermatología desde abril de 2007.

La resina epoxi se forma por polimerización de unidades que determinaran el peso molecular, y por tanto las propiedades físicas y su poder de sensibilización. Cuanto menor sea el peso molecular, mayor será el poder de sensibilización del compuesto $^{(2)}$.

En el caso de bisfenol A, el compuesto está formado por un único isómero, en contraposición a bisfenol $\mathrm{F}$, que es más complejo químicamente y está formado por tres isómeros que se encuentran en diferente proporción dependiendo de la casa comercial.

Las resinas de aplicación comercial son mezcla de polímeros de diferentes pesos moleculares y en algunos casos de diferentes compuestos epoxi, bisfenol A/ bisfenol F por lo que es importante conocer la composición exacta de los productos que manejan los trabajadores, no siempre fácil, debido a la declaración incorrecta de la fórmula por el fabricante y la capacidad de sensibilización de los diferentes alergenos que forman el producto ${ }^{(4)}$.

La capacidad de sensibilización de bisfenol $\mathrm{F}$ respecto a bisfenol A es desconocida ${ }^{(3)}$ y también la existencia de reacciones cruzadas de los diferentes isómeros de bisfenol $\mathrm{F}$ con bisfenol $\mathrm{A}$, como apuntan estudios anteriores ${ }^{(5)}$. Se han sugerido reacciones simultáneas, múltiples o cruzadas $^{(6)}$, en las que influyen factores como concentración o capacidad de penetración de las diferentes resinas.

El objetivo de nuestro estudio es conocer y comparar la incidencia de sensibilización a bisfenol A y $\mathrm{F}$, tras ser introducido este último en la batería de resinas epoxi en nuestro servicio en abril de 2007.

\section{MATERIAL Y MÉTODOS}

Se realiza un estudio descriptivo transversal en 39 trabajadores evaluados en el Servicio de Dermatología Laboral de la Escuela Nacional de Medicina del Trabajo (ENMT) durante el período de cinco años, comprendido entre 2003 y 2007.

El criterio de inclusión en este estudio es el de presentar resultado positivo a resina epoxi en la prueba epicutánea con la batería estándar.

Las pruebas epicutáneas realizadas a estos pacientes fueron la batería estándar (True test ${ }^{\circledR}$ con una serie de alergenos ampliados), batería de resinas epoxi tradicional (2003-abril 2007) y la modificada a partir de abril de 2007. También se parcheó la batería de gomas en 2 casos, según la exposición laboral. La lectura de las pruebas se realizó las 48 y 96 horas. 
Se estudian diferentes variables sociodemográficas: Sexo, edad y puesto de trabajo.

Las variables específicas de este estudio son:

- Prevalencia de sensibilización a resina epoxi 2003- 2007

- Resultados positivos de diferente alergenos de la batería estándar.

- Resultados positivos de diferentes alergenos en la batería epoxi.

- Resultado positivo a bisfenol F de la batería epoxi modificada.

- Período de sensibilización.

- Localización de las lesiones dermatológicas.

- Relevancia.

- Diagnóstico.

- Recomendación: protección, cambio de puesto de trabajo, incapacidad laboral.

\section{RESULTADOS}

Los 39 pacientes que incluye nuestro estudio se distribuyen de la siguiente manera: 35 de ellos antes de la modificación de la batería de resinas epoxi y 4 tras dicha modificación.

En estos dos grupos observamos que la edad media en el primero es de 41.01 años, con edades comprendidas entre 23 y 66 años, de los cuales 8 eran mujeres y 23 hombres. En el segundo grupo la edad media es de 57.5 años, con edades entre 51 y 66 años, y todos eran hombres. Los resultados globales muestran una edad media de 42.77 años (23-66), 27 de los cuales eran hombres y 8 mujeres.

En cuanto a la profesión los resultados obtenidos en toda la muestra son: 7 instaladores de suelos especiales (17\%), 6 pintores (15\%), 5 trabajadores de la construcción $(12 \%), 3$ operarios del sector aeronáutico $(7 \%), 2$ operarios de maquinaria y mantenimiento (5\%), 2 operarios del sector naval $(5 \%)$. Con un 2\% cada uno encontramos un mecánico ajustador de máquinas, un portero, un soldador, un montador de placas, un operario de fábrica de molinos, un mecánico de equipos electrónicos, un operario de fábrica de moquetas, una auxiliar de geriatría, un fontanero, un operario de metalurgia, un operario de fábrica de calzado, un operario de industria química, un operario de fábrica de fibra de vidrio y un artesano del caucho.

La prevalencia de sensibilización a resina epoxi fue de un $2 \%$.

Los resultados de las pruebas epicutáneas con batería estándar muestran las siguientes positividades: 6 positivos a níquel (15\%), 4 a cromo (10\%), 4 a tiomersal (10\%), 3 a cobalto $(7 \%), 2$ a mezcla de carbas $(5 \%), 2$ a tiuram mix o mezcla de tiuram $(5 \%), 1$ a antioxidantes de las gomas negras $(2 \%), 1$ a parafenilendiamina $(2 \%), 1$ a mercurio $(2 \%)$ y 1 a lanolina $(2 \%)$.

Las positividades encontradas en la batería epoxi tradicional y modificada se resumen en la tabla II.

Tabla II. Bateria epoxi y resultados positivos encontrados.

\begin{tabular}{|c|c|c|c|c|}
\hline$\overline{\text { FECHA }}$ & ALERGENO & PRE-2007 & POST-2007 & TOTAL \\
\hline \multirow[t]{9}{*}{ PRE-2007 } & 1. Hexamethilenteramina $2 \%$ & - & - & 0 \\
\hline & 2. Diaminodifenilmetano $0.5 \%$ ( DADPM) & 2 & 2 & 4 \\
\hline & 3. Trietilentetramina $0.5 \%$ (TET) & 4 & - & 4 \\
\hline & 4. Fenilglicidileter $0.25 \%$ ( PGE) & 6 & 1 & 7 \\
\hline & 5. Dietilentriamina 1\% ( DET) & 1 & - & 0 \\
\hline & 6. Isoforon Diamina $0.1 \%$ & - & - & 0 \\
\hline & 7. Resina Epoxi Cicloalifática 0.5\% ( RECA) & 1 & - & 1 \\
\hline & 8. Etilendiamina Dihidroclorhidrato $1 \%$ agua & - & - & 0 \\
\hline & 9. Dimetilamino propilamina $1 \%$ agua ( DMAPA) & 1 & - & 0 \\
\hline \multirow[t]{5}{*}{ POST-2007 } & 10. Bisfenol F 0.25\% (BF) & 3 & - & 3 \\
\hline & 11. 1,6 Hexanediol diglici dileter $0.25 \%$ & - & - & 0 \\
\hline & 12. 1,4 Butanediol diglicidileter $0.25 \%$ & - & - & 0 \\
\hline & 13. $\mathrm{m}$ - Xililendiamina $0.1 \%$ & - & - & 0 \\
\hline & 15. Tirmetilpropano triglicidileter $0.25 \%$ & - & - & 0 \\
\hline
\end{tabular}


La localización de las lesiones en el período pre2007 se agrupa en manos en 19 pacientes (54\%), brazos en 8 pacientes $(22 \%)$, cara en 6 pacientes (17\%) y cuerpo en 2 pacientes $(5 \%)$.

En el grupo post-2007 encontramos un paciente con localización de lesiones en manos $(25 \%)$ y 3 pacientes en brazos (75\%). Incluyendo todos los pacientes de nuestro estudio encontramos lesiones en mano en el $51 \%$, en brazos un $28 \%$ y en cara un $15 \%$.

Atendiendo al período de sensibilización se observa que es de 25,1 meses (0-204) para el primer grupo y de 2,25 meses $(0,5-4)$ para el segundo. De forma global el período de sensibilización es de 23,8 meses (0-204).

La relevancia de estas sensibilizaciones es actual en la mayoría de los casos, un $84 \%$. Desglosado por períodos encontramos que en el primero, 33 sensibilizaciones son de relevancia actual $(94 \%)$, en un caso pasada (2\%) y en otro desconocida (2\%). En el período post-2007 la relevancia de todas las sensibilizaciones a resina epoxi es actual.

La recomendación que se dio en el $46 \%$ de estos pacientes fue la de un cambio de puesto de trabajo.

En el grupo pre-2007 observamos 14 pacientes donde se recomienda cambio de puesto $(40 \%), 11$ protección adecuada (31\%), 3 la tramitación de una incapacidad laboral (8\%) y a 3 pacientes se le recomienda revisión por el dermatólogo. En los casos post-2007, a todos los pacientes se les recomienda cambio de puesto de trabajo.

Todos estos resultados se resumen en la tabla III.

Tabla III. Resultados.

\begin{tabular}{|c|c|c|c|c|c|c|}
\hline $\begin{array}{l}\text { NUM. DE } \\
\text { PACIENTES }\end{array}$ & EDAD/SEXO & $\begin{array}{l}\text { P.SENSIBILIZAC } \\
(\text { meses })^{1}\end{array}$ & $\begin{array}{l}\text { OTROS } \\
\text { POSITIVOS } \\
\text { ESTÁNDAR }^{2}\end{array}$ & LOCALIZACION $^{2}$ & RELEVANCIA & RECOMENDACIÓN \\
\hline 35 & $\begin{array}{l}41.01(23-66) \\
8 \mathrm{M} / 23 \mathrm{~V}\end{array}$ & $25.1(0-204)$ & $\begin{array}{l}\text { Ní (6), Cr (4), } \\
\text { Tiomersal(4), } \\
\text { Co(3), } \\
\text { Mezcla de } \\
\text { Carbas(2), } \\
\text { Tiuram } \\
\text { mix(2), } \\
\text { Gomas } \\
\text { negras (1), } \\
\text { PPDA(1), } \\
\operatorname{Hg}(1)\end{array}$ & $\begin{array}{l}\text { Manos (19), } \\
\text { Brazos (8), Cara } \\
(6), \text { Cuerpo (2) }\end{array}$ & $\begin{array}{l}33 \text { Actual } \\
1 \text { Pasada } \\
1 \text { Desconocida }\end{array}$ & $\begin{array}{l}11 \text { Protección } \\
14 \text { Cambio de puesto } \\
3 \text { Incapacidad } \\
3 \text { Dermatólogo } \\
4 \mathrm{NE}\end{array}$ \\
\hline 4 & $\begin{array}{l}57.5(51-66) \\
4 \mathrm{~V}\end{array}$ & $2.25(4-0.5)$ & Lanolina(1) & $\begin{array}{l}\text { Mano (1), Brazos } \\
\text { (3) }\end{array}$ & 4 Actual & 4 Cambio puest $\mathrm{o}$ \\
\hline 39 & $\begin{array}{l}42.77(23-66) \\
8 \mathrm{M} / 27 \mathrm{~V}\end{array}$ & $23.8(0-204)$ & & $\begin{array}{l}\text { Manos (20), } \\
\text { Brazos (11), Cara } \\
(6)\end{array}$ & 37 Actual & $\begin{array}{l}\text { Cambio } \\
\text { puesto(18),protección } \\
\text { (11),incapacidad(3) }\end{array}$ \\
\hline
\end{tabular}

1. No especificado en 3 pacientes. 2: Entre paréntesis el número de pacientes. Níquel; Cr: Cromo; Co: Cobalto; PPDA: Parafenilendiamina; Hg: Mercurio. NE: No especific

\section{DISCUSIÓN}

Las resinas epoxi (RE) tienen un amplio campo de aplicación y pueden formar parte de adhesivos, pinturas, recubrimientos aislantes, suelos, etc., lo que hace difícil imaginar un área tecnológica donde no tengan alguna aplicación. Son polímeros de mucha resistencia, termofraguables, que se tornan duros en presencia de determinados acelerantes o endurecedores. En la actualidad se tiende a denominar estos productos "sistema epoxi", ya que en general son mezclas de RE con materiales diversos como 
endurecedores, diluyentes, absorbentes, colorantes... Se pueden agrupar en varias categorías: cualquier compuesto que contenga un grupo epoxi en su molécula (RE, diluyentes y epoxi-propilclorato de amonio, EPTMAC), epiclorhidrína y bisfenol A, endurecedores y epoxi-acrilatos.

Las RE están compuestas por varios grupos químicos, pero de ellos, el 95\% son glicidil-éteres obtenidos de la reacción con la epiclorhidrina, bien con bisfenol A (diglicidil-eter de bisfenol A, DGEBA) o bien con bisfenol $\mathrm{F}$ (diglicidil-éter de bisfenol $\mathrm{F}$, DGEBF). Los principales sensibilizantes de las RE son los DGDBA y DGEBF. Las resinas basadas en bisfenol $\mathrm{F}$ son más nuevas, menos viscosas, más caras y se utilizan cuando se necesita mayor resistencia $^{(8)}$. Las RE derivadas del bisfenol A son una causa conocida desde hace tiempo de eccema de contanto alérgico profesional ${ }^{(9)}$. Sin embargo las RE basadas en bisfenol $\mathrm{F}$ son más novedosas como agentes causales de dermatitis de contacto $^{(10)}$, aunque estudios recientes muestran que pueden llegar a ser tan frecuentes como las RE de bisfenol $\mathrm{A}^{7}$. En nuestro de Servicio se incluye las RE de bisfenol $F$ en la bateria de resinas epoxi a partir de Abril de 2007.

En la tabla $\operatorname{IV}^{(9,10)}$ se resumen las características de los grupos de pacientes de los estudios previos de RE en la ENMT y se comparan con el actual. Se aprecia cómo nuestro estudio incluye un mayor número de pacientes en menor tiempo (39 en 46 meses) con respecto a los anteriores. Quizá sea debido al creciente desarrollo de las RE y sus aplicaciones en los últimos años, o bien a un aumento de la demanda de atención por parte de los trabajadores.

Tabla IV. Estudios previos de resinas epoxi en el servico de dermatología laboral de la ENMT.

\begin{tabular}{|l|l|l|l|}
\hline & $\begin{array}{l}\text { Enero de 1989 a } \\
\text { diciembre de 1992 }\end{array}$ & $\begin{array}{l}\text { Enero de 1995 a } \\
\text { abril de 2004 }\end{array}$ & $\begin{array}{l}\text { Enero de 2003 a } \\
\text { octubre de 2007 }\end{array}$ \\
\hline $\begin{array}{l}\text { Numero de } \\
\text { pacientes }\end{array}$ & 15 & 13 & 39 \\
\hline Periodo total & 48 meses & 88 meses & 46 meses \\
\hline $\begin{array}{l}\text { Incidencia } \\
\text { (pac/mes) }\end{array}$ & 0.32 & 0.15 & 0.85 \\
\hline Edad & 39.5 & 34.4 & 42.77 \\
\hline Localización & $-73 \%$ mano & $-69 \%$ facial & $-52 \%$ manos \\
$-53 \%$ facial & $-61 \%$ cara & $29 \%$ brazos \\
\hline $\begin{array}{l}\text { Periodo de } \\
\text { sensibilización }\end{array}$ & 6 meses & 8.8 meses & 23 meses \\
\hline $\begin{array}{l}\text { Segundo } \\
\text { alérgeno más } \\
\text { frecuente }\end{array}$ & Plásticos & $\begin{array}{l}\text { Gomas (Carbas y } \\
\text { Tiuram) }\end{array}$ & Níquel y cobalto \\
\hline
\end{tabular}

La edad continúa situándose, de forma similar a los estudios previos, en torno a los 40 años. El periodo de sensibilización en el grupo reciente es mayor que en los previos, 6 y 8.8 meses, frente a 23 meses. Quizá este cambio sea debido a la mejora de las medidas de protección de los trabajadores, con una disminución de la exposición a la RE y un mayor tiempo de latencia. La localización más frecuente de las lesiones pasa a ser, como ocurrió en la primera serie, las manos, en lugar de la cara. Los segundos contactantes más frecuentes en nuestro grupo de pacientes fueron el Níquel, al igual que en la población general, y el cobalto, que frecuentemente se asocia al anterior. Las gomas pasan a ser la tercera causa de positividades en estos pacientes, mientras que en el grupo anterior era la segunda causa. En el grupo actual sólo 4 de 39 pacientes tienen una sensibilización a alérgenos de gomas en la batería estándar, frente a 3 de 13 en el previo. La positividad a gomas en pacientes que manejan RE se debe al uso de medidas de protección. El hecho de que ahora las gomas se sitúen por debajo del Níquel, un contactante que suele ser epidémico, puede indicar un cambio en el hábito de protección de estos trabajadores, disminuyendo el uso de gomas y protegiéndose con otros materiales. Todos los positivos al Níquel en nuestra muestra fueron varones.

Las repercusiones para el trabajador con eccema de contacto a RE son importantes, puesto que en su 
mayoría, 18 de 39, se necesitó un cambio de puesto, $\mathrm{y}$ en 3 de ellos una incapacidad laboral.

La RE de la batería estándar es positiva en todos los pacientes de nuestro estudio, ya que fue el criterio de inclusión. La RE está formada por una mezcla de epiclorhidrina y bisfenol $\mathrm{A}^{6}$. La tabla II muestra los resultados positivos encontrados además en la batería epoxi. Llama la atención que antes de que se incluyera el bisfenol $\mathrm{F}$ en la batería epoxi, sólo 16 de 36 pacientes mostraba alguna positividad en esta batería. Desde que se ha incluido, 3 de 3 han dado positivo para bisfenol $\mathrm{F}$, además de a otros alergenos de la batería. Existe la posibilidad de que algunos de los pacientes pre-2007 con resultados negativos en la batería epoxi, estuvieran sensibilizados a bisfenol F. El Fenilglicidileter (FGE) es el alergeno más frecuente encontrado en este grupo de pacientes, aparece positivo en 7 de los 39. Se trata de un compuesto que forma uniones con las RE y acelera su proceso de endurecimient ${ }^{(6)}$. La Trietilentetramina (TET) se detecta en 4 pacientes, se trata de una poliamina alifática usada como endurecedor. Otros 4 pacientes muestran sensibilidad al Diaminodifenilmetano (DADPM), otro endurecedor ${ }^{(12)}$. Un paciente muestra positividad para la RE cicloalifática $(\text { RECA })^{(11)}$. Se trata de una resina basada en diglicidil-ésteres de ácido hexahidroftálico usada sobre todo en la fabricación de componentes electrónicos.
Existe otro paciente sensibilizado a la Dimetilamino propilamina (DMAPA), un endurecedor ${ }^{(11)}$. Por tanto, todos los pacientes que muestran algún alérgeno positivo en la batería epoxi, además del DGEBA, es a endurecedores. Son, por tanto, la segunda causa de sensibilizaciones al sistema epoxi en nuestro grupo de pacientes.

Los tres pacientes testados con la batería epoxi renovada mostraron positividad al bisfenol F. Esta es una situación en revisión actualmente ${ }^{(3,4,6,7)}$. La positividad concomitante de RE basadas en bisfenol A (positivo en la bateria estándar) con la de bisfenol $\mathrm{F}$ (en la batería epoxi) se puede deber a varios mecanismos. El primero es la sensibilización concomitante, es decir, un trabajador que manipule ambos tipos de resinas. El segundo se basa en estudios recientes que demuestran que las resinas de Bisfenol $\mathrm{F}$ son incorrectamente declaradas, y que contienen trazas de bisfenol A. Estas trazas son capaces de sensibilizar a los trabajadores, ya que el bisfenol A es un potente sensibilizante ${ }^{(4)}$. La tercera posibilidad se basa en estudios animales que demuestran la existencia de una reactividad cruzada entre ambos tipos de resinas. Dos de los tres isómeros del DGEBF reaccionan de forma cruzada con el único isómero del DGEBA (figura 1) ${ }^{(5)}$. Estos dos isómeros del DGEBF constituyen aproximadamente entre el 60 y el $100 \%$ del total y tienen potencia sen-

Figura 1. Imagen de los tres isómeros del Diglicidiléter de bisfenol $F$ (oo', op' y pp' DGEBF) y del único isómero del Diglicidiléter de bisfenol A (DGEBA) y su patrón de reacciones cruzadas (Tomado de Lee NH at al,

Contact Dermatitis 2002, 13(3): 108-115.

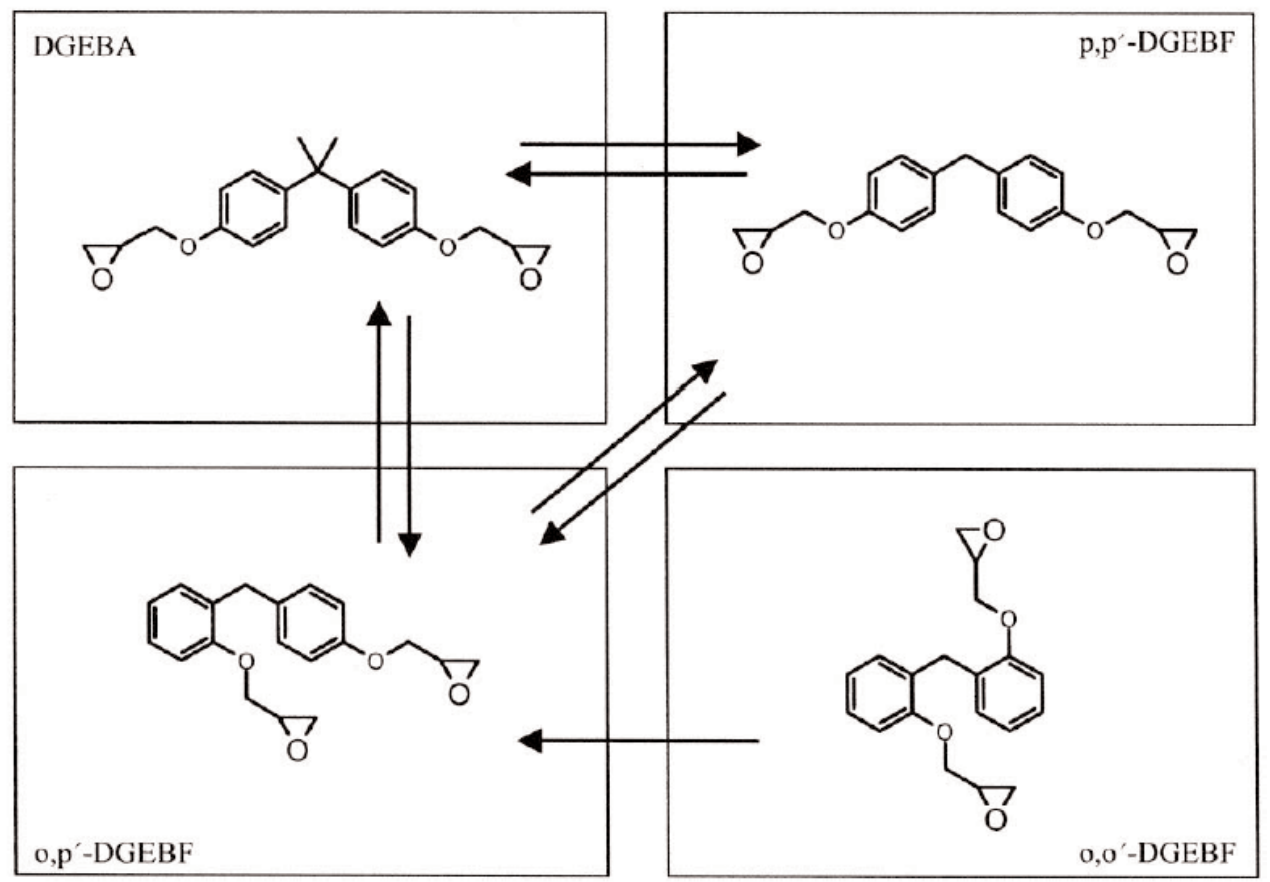


sibilizante similar al DGEBA ${ }^{(5,6)}$. Existen casos publicados de la situación contraria. Pacientes negativos para RE de bisfenol A y positivos para las de bisfenol $\mathrm{F}^{(6,7)}$. Esto puede ser debido a la sensibilización a un tipo de resina sólo de bisfenol $\mathrm{F}$, sin reactividad cruzada.

Las RE continúan siendo una causa importante de dermatitis alérgica de contacto ocupacional y de incapacidad y cambios de puestos de trabajo. De forma paralela a su desarrollo con fines industriales van apareciendo sensibilizaciones en los trabajadores que las manejan. Por otro lado, su composición

\section{BIBLIOGRAFÍA}

1. Conde-Salazar L, Ancona-Alayon. Dermatología Profesional.134-147.

2. Conde-Salazar L, Guimaraens, Romero, Harto. Resinas epoxi en la industria. Medicina y Seguridad del Trabajo XXX; 119:159-166.

3. Poten A, Bruze M. Contact allergy to epoxy resin based on diglycidyl eter of bisfenol F. Contact Derm 2001; 44: 98-99.

4. Poten A, Zimerson E, Sorensen O, Bruze M. Chemical análisis of monomers in epoxy resins based on bisphenol F and A. Contact Derm 2004; 50: 289-297.

5. Poten A, Zimerson E, Sorensen O, Bruze M. Sensitizing capacity and cross-reaction pattern of the isomers of diglycidyl ether of bisphenol $\mathrm{F}$ in the guinea pig. Contact Derm 2002; 47: 293-298.

6. Poten A, Bruze M. Occupational allergic contact dermatitis from epoxy resins base don bisphenol F. Contact Derm 1999; 41: 235.

7. Van Putten PB, Coenreaads PJ, Nater JP. Hand dermatoses and contact allergic reaction in construcción workers exposed to epoxy resins. Contact Derm 1984; 10: 146-150. a partir de una mezcla de sustancias, la demostración en diferentes trabajos de que en ocasiones son incorrectamente declaradas y el mecanismo complejo de interacciones entre bisfenol A y F, obliga a ser cauteloso a la hora de estudiar a estos pacientes. Una relevancia positiva para bisfenol A puede pasar desapercibida si sospechamos una sensibilización al bisfenol F, y viceversa, como ocurría en nuestro centro en años anteriores, antes de incluir el bisfenol $\mathrm{F}$ en la batería epoxi. Recomendamos por tanto el estudio siempre de ambos tipos de resinas en pacientes con sospecha de dermatitis alérgica de contacto por RE.

8. Bruze M, Edenholm M, Engstrom K, Svensson G. Occupational dermatosis in a Sweeden aircarft plant. Contac Derm 1996; 43: 336-340.

9. L. Conde Salazar, González de Domingo MA, Guimaraens D, Sensititazion to epoxy resin systems in special flooring workers. Contac Derm 1994; 31: 157-160.

10. Conde- Salazar L, Pastro MA, Gatica ME, Nuñez R, Henche R. Sensibilización a los sistemas de resinas epoxi en una nueva serie de pacientes instaladores de suelos especiales. Actas Dermosifiliogr 2003; 94: 144-149.

11. Chemotechnique Diagnostics: Catalogue 2006. Ed JMS Mediasystem. Modemgatam ( Sweden). 2006.

12. Conde-Salazar Gómez L, Valks R. Dermatosis profesionales por plásticos y resinas, p 134-147. En Conde-Sálazar L, Ancona- Alayón A. Dermatología Profesional. Ed Aula Médica, Madrid, 2004. 